\title{
A family of non-invertible prime links: Corrigendum
}

\author{
James M. McPherson
}

Wilbur Whitten has pointed out to me that there exist invertible links amongst my family of allegedly non-invertible links [3], and a letter from Neville Smythe has confirmed that the error lies in the third paragraph from the bottom of page 106. Professor Smythe also suggested that the use of F-isotopy was possibly a red herring and, accordingly, I have devised a simpler proof of the existence of infinitely many non-invertible prime links in $S^{3}$ (although Wilbur Whitten's paper [4] makes such a proof redundant).

Let $k_{1}$ be a non-invertible pretzel knot, and let $V$ be a tame closed regular neighbourhood of $k_{1}$. There exists a simple closed curve $k_{2}$ on $\mathrm{Bd} V$ such that $k_{1}$ and $k_{2}$ together bound a nonsingular annulus in $V$ ([2], p. 26). The link $k_{1} \cup k_{2}$ is therefore of genus zero, and so is prime if it is not geometrically splittable ([1], Theorem 3). $k_{1} \cup k_{2}$ is certainly non-invertible.

Suppose that $k_{1} \cup k_{2}$ was splittable, and let $C$ be the class of all 3-cells in $S^{3}-k_{1}$ which contain $k_{2}$ in their interiors. There exists a 3 -cell $C \in C$ such that

(i) $\mathrm{Bd} C \cap \mathrm{Bd} V$ consists of finitely many simple closed curves which are not tangent curves, and

(ii) the number of curves of $\mathrm{BdC} \cap \mathrm{BdV}$ is minimal for all 3-cells in $C$.

Suppose some intersection curve bounds a disc $D^{*}$ on $B d V$. Then $D^{*}$ contains a subdisc $D$ which is bounded by an intersection curve $\alpha$, and

Received 10 March 1971. The author would like to thank Professors Whitten and Smythe for their correspondence. 
which contains no intersection curves in its interior. Because $k_{2}$ lies in IntC, $k_{2} \cap \mathrm{Bd} D=\emptyset$; because $k_{2}$ lies on $\mathrm{Bd} V$ but is not contained in any disc on $\operatorname{BdV}\left(H_{1}(V)\right.$ is generated by $\left.k_{2}\right)$, it follows that $k_{2} \subset \operatorname{Ba} V-D$. Then if $D \subset C$, one component, $C^{\prime}$ say, of $C$ - \{an open regular neighbourhood of $D\}$ contains $k_{2}$ in its interior and lies in $S^{3}-k_{1} ; C^{\prime} \in C$. If $D \subset \mathrm{Cl}\left(S^{3}-C\right)$, let $S \subset S^{3}-k_{2}$ be the component of the 3-cell

$$
\mathrm{Cl}\left(S^{3}-C\right)-\{\text { an open regular neighbourhood of } D\}
$$

which contains $k_{1}$ in its interior; $S$ is a $3-\operatorname{cell}$, so $C^{\prime}=\mathrm{Cl}\left(S^{3}-S\right)$ is also a 3-cell, and $C^{\prime} \in C$. For both choices of $C^{\prime}$, we have

$$
\mathrm{Bd} C^{\prime} \cap \operatorname{Bd} V \subset \operatorname{Ba} C \cap \operatorname{Ba} V-\{\alpha\} .
$$

But this contradicts the minimality assumption involved in our choice of the 3-cell $C$ in $C$, so no such curve $\alpha$ can exist; that is, no curve of $\mathrm{BdC} \cap \mathrm{Bd} V$ can be nullhomologous on $\mathrm{Bd} V$.

If $B d C \cap B d V$ is not empty, therefore, there exists an intersection curve $\alpha^{\prime}$ which is not nullhomologous on $\mathrm{Bd} V$, yet bounds a disc $D^{\prime}$ on $\mathrm{BdC}$ which lies in $\mathrm{Cl}\left(S^{3}-V\right)$ and contains no intersection curves in its interior. This is impossible because $B d V$ is incompressible in $S^{3}-k_{1}$, and no such $\alpha^{\prime}$ can exist; we conclude that $B d C \cap \operatorname{Bd} V=\emptyset$.

Then either $\mathrm{BdC} \subset \operatorname{Int} V$, or $\mathrm{Bd} C \subset\left(S^{3}-V\right)$. In the first case, $\mathrm{Cl}\left(S^{3}-C\right)$ is a 3-cell (by Alexander's Theorem) which contains $k_{1}$ in its interior and itself lies in the interior of $V$ - this is impossible because $k_{1}$ has order one in $V$. In the second case, both $k_{1}$ and $k_{2}$ lie in the component of $S^{3}-\mathrm{Bd} C$ which contains $V$, so $\mathrm{Bd} C$ cannot split $k_{2}$ from $k_{1}$, as assumed.

It follows that $k_{1} \cup k_{2}$ is not geometrically splittable, and so is a prime link. Since there are infinitely many non-invertible pretzel knots, we may obtain infinitely many non-invertible prime links in this way.

\section{Peferences}

[1] Yoko Hashizume, "On the uniqueness of the decomposition of a link", Osaka Math. J. 10 (1958), 283-300. 
[2] L.P. Neuwirth, Knot groups (Annals of Mathematics Studies, Number 56; Princeton University Press, Princeton, New Jersey, 1965).

[3] James M. McPherson, "A family of non-invertible prime links", Bulz. Austral. Math. Soc. 4 (1971), 105-108.

[4] Wilbur Whitten, "On prime noninvertible links", Bulz. Austraz. Math. Soc. 5 (1971), 127-130.

School of General Studies,

Australian National University,

Canberra, ACT. 